\title{
Neurobiología del trastorno obsesivo-compulsivo: aportaciones desde la resonancia magnética funcional (II)
}

\author{
Josep Pena-Garijo, M. Ángeles Ruipérez-Rodríguez, Alfonso Barros-Loscertales
}

Introducción. En los últimos años, la neurociencia ha experimentado un creciente interés por aplicar sus métodos al conocimiento de los trastornos psiquiátricos, y una de las herramientas fundamentales para ello son las técnicas de neuroimagen. No obstante, en general, los estudios de aplicación de la resonancia magnética funcional en este ámbito son escasos y, en ocasiones, contradictorios.

Objetivo. En este trabajo se revisa la bibliografía especializada y se describe, de forma crítica, la literatura científica existente hasta la actualidad sobre la aplicación de resonancia magnética funcional y de tensor de difusión a uno de los trastornos más estudiados desde un punto de vista neurobiológico, como es el trastorno obsesivo-compulsivo.

Desarrollo. Se revisan los artículos sobre la utilización de la resonancia magnética funcional, así como aquéllos que han investigado la conectividad neural, indexados en las bases de datos médicas más utilizadas sobre el tema desde 1996.

Conclusión. La mayoría de estudios sugiere que el córtex prefrontal (orbitofrontal y cíngulo), los ganglios basales y el tálamo están relacionados con la patogénesis del trastorno obsesivo-compulsivo. Asimismo, se constatan alteraciones en la sustancia blanca que afectan a la conectividad neural. Las aportaciones de la neuroimagen en general, y de la resonancia magnética funcional en particular, son y serán, sin duda, una herramienta de especial interés para aclarar la etiología de este trastorno.

Palabras clave. Conectividad. Neuroimagen. Revisión. RMf. Tensor de difusión. Trastorno obsesivo-compulsivo.

\section{Introducción}

Desde una perspectiva diagnóstica, y desde hace más de 25 años, las técnicas de neuroimagen han entrado a formar parte del arsenal diagnóstico en medicina clínica y en la investigación biomédica, y son una herramienta de gran valor en el conocimiento tanto de la etiología, como del pronóstico y la terapéutica de los trastornos mentales [1]. En particular, la resonancia magnética funcional (RMf) es una variante de la resonancia que ofrece una mejor resolución espacial y temporal que la tomografía computarizada por emisión de fotón único o la tomografía por emisión de positrones (PET), además de su carácter no invasivo. La RMf aprovecha las propiedades magnéticas diferentes de la oxihemoglobina y la desoxihemoglobina para detectar cambios localizados en el nivel de oxigenación de la sangre, asociados de forma indirecta al nivel de activación neural de una región cerebral en la que se localizan estos cambios.

En la primera parte de este trabajo [1] se revisó el estado de la cuestión de la neuroimagen y la neuropsicología del trastorno obsesivo-compulsivo (TOC), concluyéndose que la inhibición de respuestas como mediador cognitivo y el circuito corticoes- triadotalámico, como sustrato neural, explicaban una parte importante de la etiopatogenia del TOC. En esta segunda parte vamos a revisar las aportaciones que, desde la RMf y desde los estudios de conectividad, se han venido haciendo al conocimiento de la enfermedad obsesiva.

\section{Estudios de neuroimagen con RMf}

En 1996, Breiter y Rauch [2] examinaron los puntos fuertes y las limitaciones del uso de la RMf en el estudio del TOC. Estos autores ya implicaron a los circuitos corticoestriatales y a la amígdala en la etiología del trastorno y previeron la progresiva implantación de la técnica de RMf en la psiquiatría y en las neurociencias en general.

En esta misma línea, es clásico el trabajo de Rauch et al [3] cuando revisaron la utilización del paradigma de aprendizaje implícito como una prueba de la función del circuito estriadotalámico. Estudios con PET y RMf confirmaron la hipótesis de fallo en el estriado derecho, a pesar de no haber pruebas de déficit en la ejecución de tareas. También se presentan datos preliminares con RMf que replicaron los datos del estudio original con PET en TOC y en
Departamento de Psicología Básica, Clínica y Psicobiología. Facultad de Ciencias Humanas y Sociales. Universitat Jaume I. Castellón de la Plana, España.

Correspondencia: Dr. Josep Pena Garijo. Departamento de Psicología Básica, Clínica y Psicobiología. Facultad de Ciencias Humanas y Sociales. Universitat Jaume I. Campus Riu Sec. Avda. Sos Baynat. E-12071 Castellón de la Plana.

E-mail:

jpena@psb.uji.es

Aceptado tras revisión externa: 22.02.10.

Cómo citar este artículo: Pena-Garijo J, Ruipérez-Rodríguez MA, Barros-Loscertales A. Neurobiología del trastorno obsesivo-compulsivo: aportaciones desde la resonancia magnética funcional (II). Rev Neurol 2010; 50: 541-50.

(c) 2010 Revista de Neurología 
el síndrome de Tourette, lo que sugiere la investigación de patrones de activación cerebral y su relación con las diferentes dimensiones sintomáticas.

Por último, otro trabajo de revisión que cabe destacar es el de van Veen y Carter [4], en el que intentaron probar la contribución del córtex cingulado anterior a la cognición, en situación de conflicto en el procesamiento. Analizaron varios estudios que utilizaban tareas de interferencia de respuesta y concluyeron que determinados síntomas cognitivos y conductuales que aparecen en trastornos como la esquizofrenia y el TOC pueden entenderse como resultado de una alteración en las funciones de control ejecutivo asociadas con el cíngulo anterior.

\section{Estudios que utilizan paradigmas de activación cognitiva}

\section{Córtex prefrontal}

El primer trabajo experimental que encontramos al revisar la bibliografía sobre RMf en el TOC es el de Levine et al [5], en el que utilizaron una tarea de fluencia verbal para diferenciar la ejecución en un grupo de esquizofrénicos con diferentes grados de síntomas obsesivos, medidos éstos a través de la Yale-Brown Obsessive Compulsive Scale (Y-BOCS). Observaron que se produce una mayor activación del córtex prefrontal dorsolateral izquierdo y que, al analizar la gravedad de los síntomas, éstos son inversamente proporcionales a la activación de esta área. Confirman, como otros estudios, la relación entre síntomas obsesivos en la esquizofrenia y la mayor actividad neurofisiológica. De una forma similar, el grupo de Pujol [6] estudió la activación del lóbulo frontal durante una tarea de generación de palabras en un grupo de 20 pacientes frente al mismo número de controles. Los sujetos obsesivos mostraron una mayor actividad cerebral durante la tarea y una supresión de la activación durante el período de descanso posterior. Ambas activaciones anormales correlacionaban directamente con la gravedad de los síntomas medidos a través de la Y-BOCS.

Existen otros dos estudios que confirman la implicación del córtex prefrontal en la posible patogenia del TOC, ambos utilizando tareas en las que se provoca conflicto cognitivo. Maltby et al [7], con una muestra de 14 sujetos TOC frente a otros tantos controles, mostraron, mediante la utilización de una tarea go/no-go, la activación en áreas relacionadas con el control ejecutivo y la inhibición de la acción, como son el córtex prefrontal y el cingulado anterior, así como otras áreas del circuito frontoestriado (corteza orbitofrontal lateral, caudado y tálamo), mayor en los pacientes que en los controles.
El grupo de Nakao [8] también encontró implicado al córtex prefrontal. Este estudio, además, cuenta con el mérito de ser de los pocos que utilizan un paradigma complejo en el que comparan varias condiciones experimentales y la aplicación de algún tratamiento (farmacológico o terapia de conducta). Compararon un grupo de pacientes tratados con fluvoxamina frente a un grupo tratado con terapia de conducta durante 12 semanas. En su estudio, utilizaron dos tareas experimentales: una de control cognitivo (la versión china del test de Stroop), y otra de provocación de síntomas. Tras la mejoría sintomática, al aplicar la técnica de la provocación de síntomas se producía una menor activación del córtex orbitofrontal, del prefrontal dorsolateral y del cingulado anterior. En cuanto a la aplicación del test de Stroop, se produjo un aumento en la activación de la corteza parietal y del cerebelo. La conclusión es que la mejoría clínica, tanto con fluvoxamina como con terapia de conducta, disminuye la hiperactividad del lóbulo frontal relacionada con la aparición de síntomas y aumenta la actividad del cerebro posterior relacionada con tareas de control de la acción, como el Stroop.

\section{Córtex cingulado anterior}

La intervención de la corteza cingulada en la función ejecutiva se ha contrastado ampliamente [9], así como su implicación en varios grupos de entidades psicopatológicas, como son la esquizofrenia y el TOC. En 2003, van der Wee et al [10] realizaron un estudio con pacientes sin medicación y un grupo de controles sanos, en la que midieron el rendimiento en una tarea de memoria de trabajo espacial. Los pacientes con diagnóstico de TOC sólo mostraron peor rendimiento que los controles en los niveles más altos de ejecución de la tarea, y mostraron activación en las mismas regiones cerebrales que los controles: el córtex cingulado anterior. A pesar de no disponer de evidencia suficiente, los resultados indicaron que no existía un déficit específico en la memoria de trabajo espacial, mientras que sugirieron una disfunción secundaria a otros aspectos del funcionamiento ejecutivo.

En esta misma línea, en el estudio de Nakao et al [8], citado anteriormente, los pacientes con TOC mostraban una implicación del córtex prefrontal dorsolateral y el orbitofrontal, así como una menor activación del cíngulo anterior al ejecutar un test de Stroop. Este mismo grupo [11], en otro estudio con 24 pacientes obsesivos, hallaron una menor activación del cíngulo anterior y del caudado derecho al realizar también la versión china del test de Stroop. Al igual que en otros estudios, tampoco encontra- 
ron diferencias en las puntuaciones de las pruebas neuropsicológicas. Estos datos, que parecen confirmar una hipoactividad de la corteza cingulada anterior en los pacientes con TOC, contrastan con los hallazgos de Maltby et al [7], ya que sus resultados mostraron una mayor activación del cíngulo anterior al efectuar una tarea de las denominadas de go/ no-go en pacientes con TOC. Esta mayor activación no sólo se encontró en esa región, sino en otras áreas incluidas en el circuito frontoestriadotalámico. De esta manera, si el córtex cingulado anterior desempeña un papel central en el mantenimiento de los comportamientos compulsivos, como sugieren los datos de estudios con paradigmas de control y monitorización de la acción, entonces, la activación del córtex cingulado anterior (caudal y rostral) debería producirse también en estudios de provocación de síntomas, cuestión en la que nos detendremos más adelante.

Otros estudios que aportan pruebas a favor de la hiperactividad del cíngulo anterior en relación con la aparición de síntomas obsesivos son los trabajos de Fitzgerald et al [12], que elaboraron una tarea sencilla para provocar errores, pero no síntomas obsesivos, a la que sometieron a un grupo de pacientes obsesivos $(n=8)$ y los compararon con un grupo de control $(n=7)$. Ambos grupos mostraron activación del córtex cingulado anterior durante la comisión de errores. El grupo de pacientes obsesivos mostró una mayor activación de esta área y, además, ésta era mayor cuanto mayor era la gravedad de los síntomas. Una conclusión importante se puede extraer de este estudio: la mayor actividad del córtex cingulado anterior se da en ausencia de expresión sintomática en los sujetos con trastorno obsesivo.

En esta misma línea, y confirmando la implicación del cíngulo en la etiopatogenia del TOC, se encuentra la investigación de Viard et al [13], que demostraron una mayor activación del córtex cingulado anterior y del parietal derecho en un grupo de 12 sujetos adolescentes y jóvenes con TOC de inicio en la infancia frente a un grupo de 14 controles sanos en una tarea de discriminación de estímulos visuales repetidos (control cognitivo). Este estudio concluyó también que la mayor activación del cíngulo anterior, así como de otras áreas, como la pulvinar, precuneal y paracentral, sugiere un proceso anormal en los pacientes con TOC en las tareas de conflicto y control cognitivo, y que la distribución regional de estos cambios funcionales varía con la habilidad de los pacientes para resistir las propias obsesiones. Los resultados descritos son poco concluyentes.

\section{Ganglios de la base}

Otra región clásicamente relacionada con la etiología del TOC han sido los ganglios de la base. Su implicación está demostrada en varios de los estudios que hemos mencionado anteriormente. Sin embargo, los datos son contradictorios: mientras que las investigaciones del grupo de Nakao [8] sugieren una menor activación del caudado durante la ejecución de tareas como el test de Stroop, los estudios de Maltby et al [7] apuntan a una mayor actividad del caudado durante la realización de tareas de alta conflictividad cognitiva (tarea de go/no-go), y las revisiones de Rauch et al [3] aportan datos a favor de un fallo en el estriado derecho y una disociación espacial del foco de activación del putamen y el caudado. La explicación más plausible a estas diferencias es, probablemente, la existencia de diferentes sistemas con los que están relacionadas estas estructuras y que responden diferencialmente a las distintas tareas que se implementan.

Tres estudios resumen la implicación frontoestriatal de forma contundente. En el primero, y en referencia a una tarea relacionada con la función ejecutiva, Remijnse et al [14] hallaron una actividad reducida en áreas orbitofrontales y estriatales durante la ejecución de una tarea de aprendizaje de recompensa con posterior inversión de contingencias.

En segundo lugar, la implicación del circuito frontoestriatal y su relación con la función ejecutiva en la patogenia del TOC queda avalada por el estudio de van den Heuvel et al [15]. El diseño consistió en un paradigma relacionado con eventos, utilizando una versión de la torre de Londres para comparar un grupo de 22 pacientes obsesivos libres de medicación con un grupo de 22 sujetos controles sanos. Los resultados mostraron una disfunción en la planificación (medida por el rendimiento en la torre de Londres) en el grupo de pacientes comparado con los controles. Durante la tarea se encontró en los pacientes obsesivos una disminución en la activación de las regiones frontoestriatales, principalmente en el córtex prefrontal dorsolateral y el núcleo caudado. Además, los pacientes mostraron un incremento, presumiblemente compensatorio, en áreas cerebrales que desempeñan un papel importante en la monitorización y en la memoria a corto plazo, como el córtex cingulado anterior, el prefrontal ventromedial y el parahipocampal. Estos resultados corroboran la hipótesis de un decremento en la respuesta cerebral en áreas frontoestriales, y que ésta está asociada a una disfunción en la capacidad de planificación en los pacientes obsesivos.

Muy recientemente, Roth et al [16] examinaron los sustratos neurales de la inhibición de respuesta 
en un grupo de 12 adultos frente a 13 controles a través de una tarea de go/no-go. Durante la inhibición de respuesta, el grupo de sujetos sanos mostró una mayor activación del hemisferio derecho, que incluyó el giro frontal inferior, mientras que los pacientes obtuvieron un patrón de activación más difuso. Al comparar ambos grupos, concluyeron que los pacientes afectos de TOC tienden a hipoactivar el circuito corticoestriadotalámico durante la inhibición de respuestas. También sugieren que el tálamo y los circuitos relacionados pueden desempeñar un papel en la expresión de la intensidad de los síntomas obsesivos, mientras que las regiones frontales derechas podrían estar envueltas en la supresión de síntomas.

Sin embargo, Rauch et al [17] se encontraron con un paradigma de aprendizaje implícito, en el que los pacientes obsesivos presentaron con respecto a un grupo control de sujetos sanos un funcionamiento diferente del hipocampo durante la ejecución de la tarea de aprendizaje, contrastando con la falta de replicación de la implicación del circuito frontoestriatal. A pesar de ello, las correlaciones que encontraron sugieren que esta inconsistencia con la literatura previa es atribuible a las diferencias entre las dimensiones sintomáticas del TOC.

Un apartado especial merece el estudio de Woolley et al [18], el primero que prueba la implicación frontoestrial en niños con TOC. Comparó un grupo de 10 adolescentes con diagnóstico de TOC en remisión parcial con otro de nueve sujetos sanos durante la ejecución de tres diferentes tareas de control inhibitorio. Encontraron que los adolescentes obsesivos hipoactivaban áreas del córtex orbitofrontal, tálamo y ganglios basales, demostrando una desregulación frontoestriadotalámica relacionada con el control inhibitorio en el TOC pediátrico.

\section{Estudios que utilizan paradigmas de activación mediante provocación de síntomas}

Adler et al [19] diseñaron un experimento con siete pacientes con TOC libres de medicación, a los que se midió antes y después de escanear, a través de autoinformes de síntomas obsesivo-compulsivos. Se presentaron a los sujetos imágenes diseñadas individualmente para provocar síntomas obsesivos. La presentación de estos estímulos se asoció con un aumento significativo en las escalas de síntomas autoaplicadas. Se activaron varias regiones del córtex frontal, córtex anterior, medial y lateral temporal, y cíngulo anterior derecho. La activación del frontal superior derecho correlacionó inversamente con la línea base de sintomatología compulsiva, y la activación del córtex orbitofrontal izquierdo se asoció inversamente a los cambios en los autoinformes tras los estímulos provocadores de síntomas. Estos resultados con pacientes no medicados son consistentes con los estudios previos con pacientes medicados, $y$ sugieren que los síntomas TOC están mediados por varias áreas cerebrales, que incluyen tanto el cíngulo anterior como regiones temporales y frontales.

Phillis et al [20] compararon a seis pacientes con trastorno de despersonalización, 10 pacientes con TOC y seis controles mientras visionaron fotos estandarizadas de escenas aversivas frente al visionado de fotos neutrales. Ambos grupos de control (obsesivos y normales) relataron que las fotos aversivas les produjeron mayor respuesta emocional. Respecto a los resultados del escáner, este grupo obtuvo una mayor activación en regiones relacionadas con la percepción del disgusto, la ínsula y el córtex temporooccipital que el grupo de despersonalización, que activó el córtex prefrontal ventral derecho. La ínsula sólo se activó en las escenas neutrales. La conclusión a la que llegaron es que la experiencia de carencia de emoción subjetiva en los trastornos de despersonalización se asocia a una menor respuesta en las áreas implicadas en la sensibilidad a la emoción, y un aumento en las implicadas en su regulación.

Cannistraro et al [21] por su parte, estudiaron un grupo de 10 pacientes con TOC comparándolo con un grupo de 10 controles sanos, llevando a cabo una tarea de visionado de fotos de rostros humanos que mostraban miedo o alegría y fotos neutrales. Ambos grupos activaron la amígdala para las caras de miedo frente a las neutrales. Los pacientes con TOC demostraron una menor activación que los sanos en todas las condiciones frente a la de control (un punto de fijación). Contrariamente a los demás trastornos de ansiedad, no hubo un incremento en la responsividad de la amígdala al miedo frente a la expresión neutral. Este hallazgo es consistente con el de una función anormal de la amígdala en los pacientes con TOC y podría ser un distintivo frente a los otros trastornos de ansiedad.

Schiendle et al [22] compararon un grupo de pacientes con TOC con otro de control mientras veían diferentes categorías de fotos durante una sesión de escáner RMf. El material constaba de 80 fotos estándar divididas en categorías de 'disgusto,' 'temor' y 'neutra.' Un cuarto grupo de fotos fue tomado por los propios pacientes con referencia a situaciones que les causaban específicamente fastidio o disgusto. Las fotos relevantes específicas para el TOC generaron síntomas típicos y se asociaron a una activación de la corteza frontal, la ínsula, la circunvolución supramarginal, el caudado y el tálamo. Los estímulos que causaban temor y los estímulos de disgusto no espe- 
cíicos para los pacientes también se acompañaron de activación de la ínsula, en contraste con el grupo de control y con la respuesta a los estímulos neutros de todos los grupos. Se intuye del presente estudio que los estímulos visuales que causan malestar son específicos para cada paciente. La activación de la ínsula en comparación con la amígdala sugiere que la primera procesa el disgusto y la segunda el temor.

En un estudio de Mataix-Cols et al [23] en el que se pretendía diferenciar los distintos correlatos neurales del TOC, se sometió a un grupo de 17 pacientes obsesivos y a otros tantos controles a cuatro experimentos con RMf. Se escaneó a todos los sujetos mientras visionaban bloques alternativos de escenas relacionadas con sus síntomas (contaminación, comprobación y acumulación) y escenas aversivas provocadoras de ansiedad, pero no de síntomas obsesivos. Los resultados fueron consistentes con la literatura previa en cuanto a la implicación del circuito frontoestriadotalámico. Los análisis posteriores mostraron distintos patrones de activación asociados con cada dimensión sintomática: mayor activación en los pacientes que en los controles en el córtex prefrontal ventromedial y el caudado derecho para los síntomas relacionados con la contaminación; putamen, globo pálido, tálamo y córtex dorsal para el checking; y giro precentral izquierdo y córtex orbitofrontal derecho para acumulación. Las escenas aversivas correlacionaron con mayor activación en regiones temporooccipitales. Otro dato interesante que apareció en este estudio es que no se encontraron diferencias al comparar pacientes 'puros' con pacientes con diagnósticos comórbidos.

También en el caso de los estudios con provocación de síntomas existen discrepancias en los resultados y en sus interpretaciones. No obstante, las investigaciones que hemos revisado y los estudios más recientes que incluyen modelos dimensionales [23] confirman la implicación del circuito frontoestriadotalámico, pero sugieren la existencia de correlatos neurales distintos y parcialmente superpuestos de los diferentes patrones sintomáticos del TOC.

\section{Estudios de conectividad}

Los modelos neurobiológicos del TOC ponen el énfasis en el trastorno de la función y de la conectividad de las redes corticoestriadotalámicas. Aunque los estudios de neuroimagen han ido confirmando este modelo, muy pocos estudios han llevado a cabo medidas que fueran sensibles a la conectividad cerebral en términos estructurales [24].

Las imágenes de tensor de difusión (DTI, del inglés diffusion tensor imaging) representan una téc- nica in vivo que se puede utilizar para examinar la microestructura de la sustancia blanca en seres humanos midiendo la coherencia y dirección de los tractos de fibras neurales [25]. Esta técnica permite la cuantificación de la direccionalidad y coherencia de la difusión de agua mediante índices basados en la atenuación de la señal de resonancia. Estos índices incluyen una medida de la magnitud de la difusión (MD, del inglés mean diffusion), la anisotropía del tejido (FA, del inglés fractional anisotopy) y de la orientación de la anisotropía (PDD, del inglés principal diffusion direction). Los tejidos con una alta concentración de fibras regulares tienen una alta anisotropía, mientras que aquéllos que tienen una menor regularidad, como la sustancia gris, tienen una anisotropía menor, siendo este factor el que afecta mayormente a las medidas de FA y PDD. La FA es una medida de la anisotropía en un vóxel. La PDD es una medida basada en el eje de mayor direccionalidad de la anisotropía, es decir, una estimación de la direccionalidad en un eje en particular. La medida de la alteración patológica en el tejido por DTI se traduce en valores menores de anisotropía, lo que se interpreta como una pérdida de conectividad o integridad (regularidad) de la sustancia blanca y valores menores en FA o PDD, siendo la primera la medida más extendida. La MD es una medida de movilidad de las moléculas de agua en un tejido en todas las direcciones, es decir, independientemente de la dirección de sus fibras, y que se ve afectado por el tamaño de las células y su integridad. Esta medida está siendo mucho menos aplicada. Además, la integridad de un tejido se manifiesta en valores opuestos para los índices de FA (PDD) y MD, y valores bajos de FA (PDD) y altos en MD son índices de alteración [26]. En resumen, esta técnica tiene importancia para entender la organización e integridad anatómica de la sustancia blanca y representa una herramienta importante para el estudio de la conectividad anatómica del cerebro humano [27].

White et al [25] revisaron los estudios llevados a cabo con esta técnica en los diferentes trastornos psiquiátricos. Existe una enorme heterogeneidad en los resultados obtenidos con DTI. La mayor parte de los estudios tiene que ver con la esquizofrenia y ha encontrado defectos en áreas cerebrales como el cíngulo o el cuerpo calloso, y en la sustancia blanca de regiones frontales y temporales. Las investigaciones en otros trastornos, como el TOC, la depresión o el estrés postraumático han encontrado áreas que se superponen con las encontradas en la esquizofrenia.

Una de las áreas más estudiada en este sentido ha sido el cíngulo. Szeszko et al [27] probaron la implicación de la sustancia blanca de la corteza cingulada 
anterior en la patofisiología del TOC al comparar un grupo de 15 enfermos obsesivos con un grupo de 15 sujetos sanos utilizando DTI. Los pacientes resultaron tener una menor FA en la sustancia blanca de tres áreas del giro cingulado anterior. Estos resultados, aunque preliminares, evidencian alteraciones en la sustancia blanca del cíngulo anterior. Estas alteraciones se relacionan con la patogénesis del TOC y son consistentes con los modelos neurobiológicos que postulan un defecto en la conectividad del circuito corticoestriadotalámico. Aunque el cíngulo se ha visto implicado en el TOC a lo largo de bastantes estudios, los resultados han sido contradictorios. En el estudio anterior apareció disminución de la FA en el haz cingulado izquierdo con respecto al grupo de control, mientras que el estudio de Cannistraro et al [28] demostró un aumento de la FA en la misma zona. Estos estudios también encontraron una FA aumentada en la cápsula interna y en el cuerpo calloso, y disminuida en los lóbulos parietales, el giro supramarginal y el giro lingual izquierdo. Yoo et al [29], además, demostraron que la FA había disminuido tras 12 semanas de tratamiento con citalopram. Estos resultados proveen evidencia preliminar de una arquitectura anormal del haz cingulado y del extremo anterior de la cápsula interna. Las diferencias de FA encontradas en estas áreas son consistentes con la presencia de conexiones alteradas entre las áreas conectadas por estos tractos. Este hecho podría explicar por qué intervenciones quirúrgicas sobre estos tractos son terapéuticas [29].

El cuerpo calloso es una gran estructura interhemisférica de fibras de sustancia blanca que conecta ambos hemisferios cerebrales y que tiene un papel crucial en los procesos cognitivos y de conectividad interhemisférica [30]. Por otro lado, la corteza orbitofrontal es un punto de interfaz entre la corteza de asociación, las estructuras límbicas y las regiones subcorticales implicadas en el control de las vías efectoras automáticas y motoras [31]. Aunque la investigación reciente se ha focalizado en la circuitería orbitofrontal, las anormalidades en la sustancia blanca de esta región apenas se han estudiado. Saito et al [30] sugirieron que el TOC está asociado a defectos en la integridad de la sustancia blanca de la región orbitofrontal. El propósito de su estudio fue examinar las anormalidades en la microestructura de la sustancia blanca del cuerpo calloso en pacientes obsesivos comparados con un grupo de sujetos normales, e investigar las relaciones entre las medidas de DTI en esta área y los síntomas obsesivocompulsivos. Encontraron una disminución significativa de la anisotropía en el rostrum del cuerpo calloso en el grupo de pacientes comparado con el de controles. Además, esta reducción se correlacionó con puntuaciones más bajas en la Y-BOCS. Concluyeron que esta región de la corteza prefrontal orbital está relacionada con la patofisiología del TOC y sugirieron que el circuito orbitofrontal influye directamente en la gravedad de los síntomas.

Por otra parte, Menzies et al [32] usaron DTI para medir la FA de la sustancia blanca en 30 pacientes con TOC, 30 familiares de primer grado no afectados y 30 sujetos sanos. Los pacientes mostraron una menor FA en una amplia región de la sustancia blanca inferoparietal derecha y un aumento significativo de FA en la región frontal medial derecha. Los familiares también exhibieron estas anomalías. Los autores sugieren la posibilidad de que nos encontremos ante un fenotipo de TOC relacionado con la sustancia blanca.

El estudio más reciente realizado hasta la fecha es el de Garibotto et al [33], en el que evaluaron la organización y direccionalidad de los haces de fibras mayores en un subgrupo de pacientes con TOC que incluían lavadores y comprobadores, y que presentaban déficit en tareas de toma de decisiones, utilizando las medidas de FA y de PDD en 15 pacientes frente a 16 controles. El grupo de pacientes mostró una menor FA y una PDD alterada a lo largo del cuerpo calloso, cíngulo, fascículo longitudinal superior y fascículo frontooccipital inferior, de forma bilateral. La tractografía del fascículo frontooccipital confirmó una reducción bilateral significativa de la FA. Varios parámetros correlacionaron con la gravedad de los síntomas y la ejecución neuropsicológica. Los autores concluyeron que su estudio revelaba anormalidades específicas en la sustancia blanca, lo que sugería una desorganización de los tractos neurales, reflejada a través de los cambios en la dirección de las fibras. De todo ello se concluye que esta anormalidad en la conectividad anatómica podría desempeñar un papel específico en la fisiopatología del trastorno.

Por último, tres estudios investigan la conectividad en el TOC por otras técnicas de RM. Kitamura et al [34], utilizando la espectroscopia por RM, concluyen que la sustancia blanca parietal está implicada en el origen y desarrollo del TOC. Duran et al [35] utilizaron la morfometría a través de RM para evaluar las potenciales diferencias en el volumen de la sustancia blanca de áreas como el haz cingulado y la cápsula interna en pacientes con síntomas obsesivos frente a sujetos sanos. Encontraron una reducción global del volumen de sustancia blanca en los enfermos comparados con los controles. No encontraron, sin embargo, diferencias regionales en las áreas que pretendían estudiar. No obstante, se 
encontraron grandes clusters de correlación positiva con las puntuaciones de gravedad de los síntomas en la cápsula interna, de forma bilateral. Sus hallazgos apuntan en la misma dirección que los anteriores: un defecto en la conectividad de los circuitos estriadocorticotalámicos. El último trabajo publicado del que tenemos noticia, llevado a cabo en nuestro ámbito geográfico por Harrison et al [24], utiliza la RMf para probar la hipótesis de que el TOC está asociado con alteraciones en la conectividad funcional de las regiones corticoestriatales ventrales. A pesar de que el estudio utiliza RMf, hemos considerado más adecuado incluirlo en este apartado, ya que su propósito es el estudio de la conectividad. Los autores midieron la conectividad en cuatro regiones del estriado: caudado dorsal, caudado ventral/núcleo accumbens, putamen dorsal y putamen ventral en dos grupos de sujetos (21 pacientes obsesivos frente a 21 controles sanos). En ambos grupos se encontró un patrón claro de conectividad coherente con la segregación de las redes corticoestriatales motora, asociativa y límbica. Encontraron también diferencias en la conectividad de estos patrones entre pacientes y controles. El estudio apoya la hipótesis de la alteración de las redes corticoestriatales y, específicamente, de la conectividad funcional de las regiones corticoestriatales ventrolímbicas en pacientes afectos de TOC.

En resumen, parece haber evidencia de una alteración en las redes neurales corticoestriadotalámicas no sólo a nivel estructural y funcional, sino a nivel de conectividad neural. La sustancia blanca orbitofrontal, el cíngulo, la cápsula interna y el cuerpo calloso parecen ser las áreas mayormente halladas en la investigación reciente. El fascículo frontooccipital se encuentra también alterado. Todo ello apunta a una desorganización de la conectividad anatómica en el TOC. La importancia de los estudios de tractografía que incluyan los cambios potenciales en la sustancia blanca que une y sostiene las regiones cerebrales implicadas en el TOC los convierte en un objetivo futuro de investigación en el campo de la fisiopatología de las enfermedades mentales en general, y del TOC en particular.

\section{Conclusiones}

El objetivo de este trabajo ha sido intentar resumir el estado de la cuestión de las bases neurales del TOC, sobre todo en lo que respecta a las aportaciones que, desde la RMf, se han hecho al conocimiento de la etiopatogenia de este síndrome. La bibliografía revisada dista de ser concluyente. Existen todavía numerosas discrepancias en la conceptualización de un modelo neurobiológico unitario del TOC [36].

Los sistemas cerebrales frontoestriatales implicados en el TOC incluyen el córtex orbitofrontal, el estriado y el cingulado anterior, entre otros [1].

Estudios con PET comunican un metabolismo anormal del córtex orbitofrontal en estudios de reposo.

Los estudios con RMf que han investigado la función ejecutiva también han identificado diferencias en áreas frontoestriatales en pacientes con TOC, sobre todo el córtex prefrontal y el núcleo caudado.

Asimismo, hay pruebas de una anormal actividad frontoestrial usando paradigmas de provocación de síntomas. Incluso hay ejemplos de estudios que han puesto de manifiesto cambios comparativos en regiones que no han sido de interés en estudios previos, como actividad disminuida en el córtex prefrontal dorsolateral o aumentada en el córtex parietal.

Otros autores han encontrado hiperactividad en el córtex cingulado, tanto anterior como posterior, en tareas de go/no-go, mientras que otros autores han encontrado actividad disminuida en estas mismas áreas.

El trabajo reciente de Roth et al [16] muestra que los sujetos obsesivos presentan un patrón de hipoactivación del circuito frontoestriadotalámico relacionado con la inhibición de respuesta.

Trabajos como el de Menzies et al [37], con RM estructural, parecen confirmar los hallazgos previos del mismo grupo [38] sobre el papel central del déficit en la inhibición de respuesta como mecanismo cognitivo básico en la formación de síntomas obsesivos y su correspondiente sustrato neural: el sistema frontoestriadotalámico. Sin embargo, estos autores sugieren la existencia de otros sistemas cerebrales implicados, más allá del frontoestriadotalámico.

Al hilo de lo anterior, el mismo grupo [39] identificó una activación reducida de varias regiones corticales, incluyendo el córtex orbitofrontal lateral, durante la realización de una tarea de aprendizaje inverso, tanto en el grupo de obsesivos como en sus familiares próximos no afectados por la enfermedad. El córtex orbitofrontal es también una región neural que facilita la flexibilidad conductual después de un feedback negativo (aprendizaje inverso). Todo ello lleva a pensar en otro posible marcador neurocognitivo endofenotípico que merece la pena estudiar.

Los hallazgos relacionados con la hipoactivación de las vías frontoestriatales durante la realización de tareas cognitivas en los pacientes con TOC contrastan con la hiperactivación de las mismas regiones encontradas en los estudios con paradigmas de provocación de síntomas [40]. Se ha argumentado que 
la sobreactivación del circuito frontoestriadotalámico, que es responsable de los síntomas obsesivos y compulsivos, acabaría provocando que los recursos de las regiones cerebrales implicadas en estas vías estuvieran menos disponibles para funciones cognitivas, lo que explicaría la hipoactivación concurrente de las vías frontoestriatales relacionadas con los síntomas durante la actividad cognitiva [18]. Ello se encuentra en la línea del estudio de Nakao et al [8], en el que el tratamiento farmacológico de los pacientes obsesivos condujo a una reducción de la activación frontoestriatal previamente incrementada en tareas de provocación de síntomas, mientras que aumentó la previamente reducida activación frontal durante una tarea cognitiva, como el test de Stroop.

Finalmente, los estudios de conectividad, sobre todo a través de DTI han encontrado alteraciones en la sustancia blanca orbitofrontal, el cíngulo, la cápsula interna y el cuerpo calloso. El fascículo frontooccipital se encuentra también alterado. Todo ello apunta a una desorganización de la conectividad anatómica en el TOC.

En cualquier caso, la aparente incongruencia y la variedad de hallazgos que hemos encontrado en esta revisión podrían entenderse mejor si tenemos en cuenta las limitaciones de algunos trabajos. En primer lugar, las muestras que se han utilizado en muchos casos son pequeñas. Además, en la mayoría de ellos no se han tenido en cuenta las diferentes dimensiones sintomáticas que varios autores han encontrado en el TOC, y que se corresponden con distintos patrones neurales parcialmente superpuestos [23,41]. Sería también deseable examinar la superposición de estos trastornos con otros del espectro obsesivo y de ansiedad, ya que también se ha observado que implican sustratos neurales comunes y diferentes [23]. En segundo lugar, muchos de los pacientes estudiados presentaban comorbilidad con otros trastornos. Esta condición, por otra parte muy habitual en la clínica, plantea la necesidad de tomar los datos con precaución, dada la imposibilidad de asegurar que las diferencias encontradas se deban exclusivamente a la presencia de la enfermedad obsesiva. Aun así, estudios anteriores, como el de Mataix-Cols et al [23], no encontraron diferencias en patrones de activación cerebral dentro del grupo de obsesivos, al comparar aquéllos con diagnóstico de TOC 'puro' con aquéllos que presentaban diagnósticos comórbidos. Por último, en muchos de los estudios revisados, dentro del grupo de pacientes los hay tanto medicados como libres de medicación. Este hecho, aunque ha habido autores que han demostrado que los hallazgos en ambos grupos son compatibles [19], enturbia los resultados. Próximos estudios deberán tener en cuenta ambos subgrupos de pacientes y establecer diferencias entre ellos y con un grupo control. Este último caso tendría, además, una importante relevancia clínica para constatar la efectividad de las diferentes intervenciones, tanto farmacológicas como cognitivo-conductuales, en el tratamiento de esta enfermedad.

La mayoría de estudios sugiere que el córtex prefrontal (orbitofrontal y cíngulo), los ganglios basales y el tálamo están relacionados con la patogénesis del TOC. Existe, sin embargo, cierta dificultad para integrar los hallazgos de la psicología y de las neurociencias. La investigación reciente en el campo de la neurociencia cognitiva de la función normal de estas áreas cerebrales demuestra el papel del córtex orbitofrontal en el procesamiento de la recompensa, del cíngulo anterior en la detección de errores, de los ganglios basales en la activación de programas motores y conductuales, y del córtex prefrontal en el almacenamiento de memorias de secuencias de conductas [42]. Huey et al [42] proponen un modelo explicativo de los síntomas específicos e integran los hallazgos de la psicología y la neuroanatomía.

No obstante, las aportaciones de la neuroimagen en general, y de la RMf en particular, son y serán, sin duda, una herramienta de especial interés para aclarar estas relaciones entre conducta, cognición y neurobiología, sin cuya integración de hallazgos nunca podremos tener un modelo etiopatogénico adecuado del TOC y de su espectro sintomático.

\section{Bibliografía}

1. Pena-Garijo J, Ruipérez-Rodríguez MA, Barros-Loscertales A. Neurobiología del trastorno obsesivo-compulsivo: aportaciones desde la resonancia magnética funcional (I). Rev Neurol 2010; 50: 477-85.

2. Breiter HC, Rauch SL. Functional MRI and the study of OCD: from symptom provocation to cognitive-behavioral probes of cortico-striatal systems and the amygdale. Neuroimage 1997; 4: 127-38.

3. Rauch SL, Whalen PJ, Curran T, Shin LM, Coffey BJ, Savage $\mathrm{CR}$, et al. Probing striato-thalamic function in obsessivecompulsive disorder and Tourette syndrome using neuroimaging methods. In Cohen DJ, Goetz CG, Jankovic J, eds. Tourette syndrome. Philadelphia: Lippincott Williams \& Wilkins; 2001. p. 207-24.

4. Van Veen V, Carter CS. The anterior cingulate as a conflict monitor: fMRI and ERP studies. Physiol Behav 2002; 77: 477-82.

5. Levine JB, Gruber SA, Vaird AA, Yurgelun-Todd D. Obsessive-compulsive disorder among schizophrenic patients: an exploratory study using functional magnetic resonance imaging data. Compr Psychiatry 1998; 39: 308-11.

6. Pujol J, Torres LL, Deus J, Cardoner N, Pifarré J, Capdevila A, et al. Functional magnetic resonance imaging study of frontal lobe activation during word generation in obsessivecompulsive disorder. Biol Psychiatry 1999; 45: 891-7.

7. Maltby N, Tolin DF, Worhunsky P, O'Keefe TM, Kiehl KA. Dysfunctional action monitoring hiperactivates frontalstriatal circuits in obsessive compulsive disorder: an eventrelated fMRI study. Neuroimage 2005; 24: 495-503. 
8. Nakao T, Nakagawa A, Yoshiura T, Nakatani E, Nabeyama $\mathrm{M}$, Yoshizato, et al. Brain activation of patients with obsessive-compulsive disorder during neuropsychological and symptom provocation tasks before and after symptom improvement: a functional Magnetic Resonance Imaging Study. Biol Psychiatry 2005; 57: 901-10.

9. Friedlander L, Desrocher M. Neuroimaging studies of obsessive-compulsive disorder in adults and children. Clin Psychol Rev 2006; 26: 32-49.

10. Van der Wee NJA, Ramsey NF, Jansma JM, Denys DA, Van Megen HJGM, Westenberg HMG, et al. Spatial working memory deficits in obsessive compulsive disorder are associated with excessive engagement of the medial frontal cortex. NeuroImage 2003; 20: 2271-80.

11. Nakao T, Nakagawa A, Yoshiura T, Nakatani E, Nabeyama $\mathrm{M}$, Yoshizato C, et al. A functional MRI comparison of patients with obsessive-compulsive disorder and normal controls during a Chinese character Stroop task. Psychiatry Res 2005; 139: 101-14.

12. Fitzgerald KD, Welsh RC, Gehring WJ, Abelson JL, Himle JA, Liberzon I, et al. Error-related hyperactivity of the anterior cingulate cortex in obsessive-compulsive disorder. Biol Psychiatry 2005; 57: 287-94.

13. Viard A, Flament MF, Artiges E, Dehaene S, Naccache, Cohen $D$, et al. Cognitive control in childhood-onset obsessivecompulsive disorder: a functional MRI study. Psychol Med 2005; 35: 1007-17.

14. Remijnse PL, Nielen MA, Van Balkom AJ. Reduced orbitofrontal-striatal activity on a reversal learning task in obsessivecompulsive disorder. Arch Gen Psychiatry 2006; 63: 1225-36.

15. Van den Heuvel OA, Veltman DJ, Groenewegen HJ, Catz DC, Anton JLM, Van Balkom AJ, et al. Frontal-striatal dysfunction during planning in obsessive-compulsive disorder. Arch Gen Psychiatry 2005; 62: 301-10.

16. Roth RM, Saykin AJ, Flashman LA, Pixley HS, West JD, Mamourian AC. Event related functional magnetic resonance imaging of response inhibition in obsessivecompulsive disorder. Biol Psychiatry 2007; 62: 901-9.

17. Rauch SL, Wedig MM, Wright CI, Martis B, McMullin KG, Shin LM, et al. Functional magnetic resonance imaging study of regional brain activation during implicit sequence learning in obsessive-compulsive disorder. Biol Psychiatry 2007; 61: 330-6.

18. Woolley J, Heyman I, Brammer M, Frampton I, McGuire PK, Rubia K. Brain activation in paediatric obsessivecompulsive disorder during tasks of inhibitory control. Br J Psychiatry 2008; 192: 25-31.

19. Adler CM, McDonough-Ryan P, Sax KW, Holland SK, Arndt S, Strakowski SM. fMRI of neuronal activation with symptom provocation in unmedicated patients with obsessive compulsive disorder. J Psychiatr Res 2000; 34: 317-24.

20. Phillis ML, Medford N, Senior C, Bullmore ET, Suckling J, Brammmer MJ, et al. Depersonalization disorder: thinking without feeling. Psychiatry Res 2001; 108: 145-60.

21. Cannistraro PA, Wright CI, Weddig MM, Martis B, Shin LM, Wilhelm S, et al. Amygdala responses to human facies in obsessive-compulsive disorder. Biol Psychiary 2004; 56: 916-20.

22. Schiendle A, Schäfer A, Stark R, Walter B, Vaitl D. Neural responses of OCD patients towards disorder-relevant, generally disgust-inducing and fear-inducing pictures. Int J Psychophysiol 2005; 57: 69-77.

23. Mataix-Cols D, Wooderson S, Lawrence N, Brammer MJ, Speckens A, Phillips ML. Distinct neural correlates of washing, checking, and hoarding symptom dimensions in obsessive-compulsive disorder. Arch Gen Psychiatry 2004; 61: 564-76.

24. Harrison BJ, Soriano-Mas C, Pujol J, Ortiz H, López-Solá M, Hernández-Ribas R, et al. Altered corticostriatal functional connectivity in obsessive-compulsive disorder. Arch Gen Psychiatry, 2009; 66: 1189-200.

25. White T, Nelson M, Lim KO. Diffusion tenor imaging in psychiatric disorders. Top Magn Reson Imaging, 2008; 19: 97-109.

26. LeBihan D, Margin JF, Poupon C, Clark CA, Pappata S, Molco N, et al. Diffusion tensor imaging: concepts and applications. J Magn Reson Imaging 2001; 13: 534-46.

27. Szeszko PR, Ardekani BA, Ashtari M, Malhotra AK, Robinson DG, Bilder RM, et al. White matter abnormalities in obsessive-compulsive disorder: a diffusion tensor imaging study. Arch. Gen. Psychiatry 2005; 62: 782-90.

28. Cannistraro PA, Makris N, Howard JD, Wedig MM, Hodge SM, Wilhelm S, et al. A diffusion tensor imaging study of white matter in obsessive-compulsive disorder. Depress Anxiety 2007; 24: 440-6.

29. Yoo SY, Jang JH, Shin YW, Kim DJ, Park HJ, Moon WJ, et al. White matter abnormalities in drug-naïve patients with obsessive-compulsive disorder: a diffusion tensor study before and after citalopram treatment. Acta Psychiatr Scand 2007; 116: 211-9.

30. Saito Y, Nobuhara K, Okugawa G, Takase K, Subimoto $\mathrm{T}$, Horiuchi M, et al. Corpus callosum in patients with obsessive-compulsive disorder: diffusion-tensor imaging study. Radiology 2008; 246: 536-42.

31. Zald DH, Kim SW. Anatomy and function of the orbital frontal cortex I. Anatomy, neurocircuitry, and obsessive-compulsive disorder. J Neuropsyciatry Clin Neurosci 1996; 111: 209-19.

32. Menzies L, Williams GB, Chamberlain SR, Ooi C, Fineberg $\mathrm{N}$, Suckling J, et al. White matter abnormalities in patients with obsessive-compulsive disorder and their first-degree relatives. Am J Psychiatry 2008; 165: 1308-15.

33. Garibotto V, Scifo P, Gorini A, Alonso CR, Brambati S, Bellodi L, et al. Disorganization of anatomical connectivity in obsessive compulsive disorder: a multi-parameter diffusion tensor imaging in a subpopulation of patients. Neurobiol Dis 2010; 37: 468-76.

34. Kitamura H, Shioiri T, Kimura T, Ohkubo M, Nakada T, Someya T. Parietal white matter abnormalities in obsessivecompulsive disorder: a magnetic resonance spectroscopy study at 3-Tesla. Acta Psychiatr Scand 2006; 114: 101-8.

35. Duran FL, Hoexter MQ, Valente AA Jr, Miguel EC, Busatto GF. Association between symptom severity and internal capsule volume in obsessive-compulsive disorder. Neurosci Lett 2009; 452: 68-71.

36. American Psychiatric Association. Manual diagnóstico y estadístico de los trastornos mentales, 4 ed., texto revisado (DSM-IV-TR). Barcelona: Masson; 2000.

37. Menzies L, Achard S, Chamberlain SR, Fineberg N, Chen $\mathrm{CH}$, Del Campo N, et al. Neurocognitive endophenotypes of obsessive-compulsive disorder. Brain 2006; 130: 3223-36.

38. Chamberlain SR, Blackwell AD, Fineberg NA, Robbins TW, Sahakian BJ. The neuropsychology of obsessive compulsive disorder: the importance of failures in cognitive and behavioural inhibition as candidate endophenotypic markers. Neurosci Biobehav Rev 2005; 29: 399-419.

39. Chamberlain SR, Menzies L, Hampshire A, Suckling J, Fineberg NA, Del Campo N, et al. Orbitofrontal dysfunction in patients with obsessive-compulsive disorder and their unaffected relatives. Science 2008; 321: 421-2.

40. Whiteside SP, Port JD, Abramowitz JS. A meta-analysis of functional neuroimaging in obsessive-compulsive disorder. Psychiatry Res Neuroimag 2004; 132: 69-79.

41. Mataix-Cols D, Rosario-Campos MC, Leckman JF. A multidimensional model of obsessive-compulsive disorder. Am J Psychiatry 2005; 162: 2193-4.

42. Huey ED, Zahn R, Krueger F, Moll J, Kapogiannis D, Wassermann EM, et al. A psychological and neuroanatomical model of obsessive-compulsive disorder. J Neuropsychiatry Clin Neurosci 2008; 20: 390-408. 
The neurobiology of obsessive-compulsive disorder: new findings from functional magnetic resonance imaging (II)

Introduction. In recent years, neuroscience has shown a growing interest in applying its methods to furthering the knowledge of psychiatric disorders, and one of the fundamental tools used to do so are neuroimaging techniques. Yet, in general, few studies have been conducted in which functional magnetic resonance has been applied in this field and findings are sometimes contradictory.

Aims. In this study we review the specialised bibliography and present a critical discussion on the scientific literature published to date on the application of functional magnetic resonance and diffusion tensor imaging to one of the most widely studied disorders, from a neurobiological point of view, namely, obsessive-compulsive disorder.

Development. The study reviews the articles on the use of functional magnetic resonance imaging, as well as those dealing with neural connectivity, that have been indexed in the most commonly used medical databases on the topic since 1996.

Conclusions. Most studies suggest that the prefrontal cortex (orbitofrontal and cingulate), the basal ganglia and the thalamus are involved in the pathogenesis of obsessive-compulsive disorder. Likewise, alterations in the white matter that affect neural connectivity have also been found. The contributions made by neuroimaging and, more specifically, by functional magnetic resonance imaging are and will undoubtedly continue to be a particularly interesting tool for explaining the aetiology of this disorder.

Key words. Connectivity. Diffusion tensor imaging. fMRI. Neuroimaging. Obsessive-compulsive disorder. Review. 